\title{
Heartbeat: Rule-out of acute myocardial infarction - risky business
}

For as many as 1 in 10 patients presenting to hospital emergency departments, chest pain is the cause for evaluation. However, cardiac ischemia is the underlying etiology of chest pain in fewer than $20 \%$ of these emergency department patients. The rapid and accurate diagnosis of myocardial injury is central to the early and safe discharge of patients with non-life threatening causes of chest pain.

The advent of high-sensitivity cardiac troponin assays (hs-cTn) has facilitated the detection of low levels of myocardial injury, and potentially a more rapid diagnosis of myocardial injury among chest pain patients. Similarly, the sensitivity of these assays may permit more rapid exclusion of myocardial injury as the etiology of chest pain. Accordingly, the European Society of Cardiology (ESC) and National Institute for Health Care Excellence (NICE) guidance suggests rule-out of acute myocardial infarction based on hs-cTn levels at 3 hours after presentation.

The article by Pickering, et al. (see page 1270) sought to assess the validity of the ESC guidance on ruling-in and ruling-out acute myocardial infarction based on hs-cTn levels on presentation and 3 hours after presentation. Among 2,046 patients with acute chest pain, the rule-out sensitivity of the ESC algorithm was 93.2\% (95\% CI $87.5 \%$ to $96.8 \%$ ) for hs-cTnI and $94.8 \%$ (95\% CI $89.5 \%$ to $97.9 \%$ ) for hs-cTnT. Modifications to the algorithm improved the sensitivity of the diagnostic algorithm to above $99 \%$ for the rule-out of acute myocardial infarction (figure 1).

In the editorial by Neumann, et al., (see page 1251) guidance is offered on how application of lower troponin cut-offs than proposed in initial diagnostic guidance statements may enhance opportunities for early discharge without increasing the number of missed myocardial infarctions.

In the article by Parsonage, et al., (see page 1279) is an assessment of the validity of the NICE recommended algorithms for the rule-out of acute myocardial infarction based on hs-cTn assays. In an evaluation of 6,502 patients drawn from 3 cohorts of patients presenting with chest pain, the

Correspondence to Professor Catherine M Otto, Division of Cardiology, University of Washington, Seattle, WA 98195, USA; cmotto@u.washington.edu overall sensitivity for myocardial infarction rule-out using hs-TnI was $89.3 \%$ (95\% CI $86.2 \%$ to $93.6 \%$ ), as compared with $98.6 \%(97.1 \%$ to $99.4 \%)$ for the hs-TnT algorithm (table 1).

The editorial by Body, et al., (see page 1253), it is noted that the NICE guidance was a technology appraisal based on economic analyses and provides insights on why further clinical guidance in the use of hs-cTn for the diagnosis of acute myocardial infarction is needed.

Throughout the work of these two manuscripts and accompanying editorials, the importance of incorporating singular test findings within the context of the entirety of patient data becomes apparent. The cohorts used in these studies were strictly defined to avoid heterogeneity in risk profiles of chest pain presentation. However, even with these restrictions, important aspects of the patient presentation, comorbidities, and risk factors may refine risk estimates for ischemic chest pain. As a result, and as noted by Parsonage, algorithms based exclusively on hs-cTn levels should not be "relied on as the sole means of excluding acute coronary syndromes in all patients presenting with chest pain," but instead should be "incorporated into a broader strategy of risk stratification and investigation that identifies these patients with unstable or prognostically important coronary artery disease."

In congenital heart disease patients with Fontan physiology, there has been considerable concern that even short term hypoxia at high altitude might be poorly tolerated. In the words of Brida and Diller (see page 1255): "Due to the passive pulmonary circulation without a subpulmonary ventricle, there is a legitimate concern that high-altitude hypoxia might induce pulmonary vasoconstriction and consequently increase pulmonary artery pressure (PAP), leading to a reduction of pulmonary blood flown(PBF) and circulatory output, both of which are already reduced at baseline in a typical Fontan patient". In order to study this question, Staempfli and colleagues (see page 1296) compared 16 adult Fontan patients to 14 matched control subjects at low and high altitude. Cardiopulmonary exercise stress testing (CPET) and measurement of PBF
Steven M Bradley, Catherine M Otto

with a gas rebreathing system were measured with 12 weeks of each other at low altitude and then at the High Alpine Research Station Jungfraujoch at $3454 \mathrm{~m}$ (a beautiful location which is well worth a visit). Although PBF was higher in controls both at rest and with exercise, there was no significant difference between low and high altitude. The reduction in peak oxygen uptake at high altitude was 9 $\pm 12 \%$ in Fontan patients compared to 17 $\pm 8 \%$, in controls $(p=0.005)$, however Fontan patients start at a lower baseline (figure 2).

Brida and Diller comment further in the accompanying editorial that: "Partly to our surprise, this study showed that there was no decrease in PBF at high altitude, both at rest and during submaximal exercise, no negative impact on exercise capacity and no adverse symptoms on short-term high-altitude exposure for Fontan patients" (figure 3). "The current

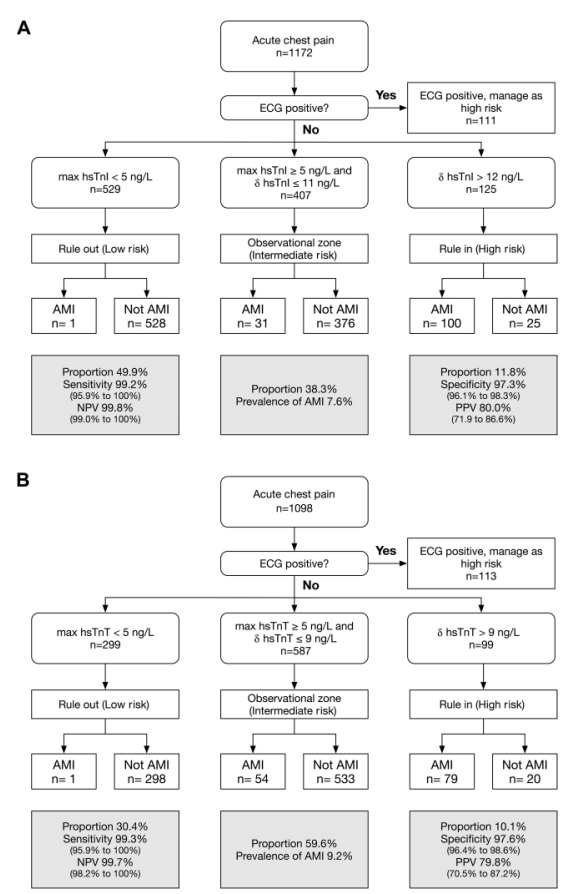

Figure 1 Simplified risk assessment strategy based on the maximum of two hs-cTn samples and the delta value of the difference between them. (A) hs-cTnl, (B) hs-cTnT. AMI, acute myocardial infarction; hs-cTnl, high-sensitivity cardiac troponin l; hs-cTnT, high-sensitivity cardiac troponin T; NPV, negative predictive value; PPV, positive predictive value. 
Table 1 Primary and secondary outcomes according to the NICE DG15 optimal algorithms for (a) the hsTnl assay and (b) the hsTnT assay

\begin{tabular}{|c|c|c|c|c|c|c|c|c|}
\hline \multirow[b]{2}{*}{ (a) } & \multicolumn{2}{|l|}{ Brisbane } & \multicolumn{2}{|l|}{ Christchurch } & \multicolumn{2}{|l|}{ Basel } & \multicolumn{2}{|l|}{ Combined } \\
\hline & $\begin{array}{l}\text { Ruled out } \\
695\end{array}$ & $\begin{array}{l}\text { Ruled in } \\
88\end{array}$ & $\begin{array}{l}\text { Ruled out } \\
719\end{array}$ & $\begin{array}{l}\text { Ruled in } \\
246\end{array}$ & $\begin{array}{l}\text { Ruled out } \\
1092\end{array}$ & $\begin{array}{l}\text { Ruled in } \\
288\end{array}$ & $\begin{array}{l}\text { Ruled out } \\
2506\end{array}$ & $\begin{array}{l}\text { Ruled in } \\
622\end{array}$ \\
\hline $\mathrm{Ml}$ at presentation & $2(0.3 \%)$ & $39(44.3 \%)$ & $12(1.7 \%)$ & $195(79.3 \%)$ & $37(3.4 \%)$ & $193(67.0 \%)$ & $51(2.0 \%)$ & $427(68.6 \%)$ \\
\hline 30-day MACE & $25(3.6 \%)$ & $42(47.7 \%)$ & $86(12.0 \%)$ & $200(81.3 \%)$ & $156(14.3 \%)$ & $215(74.6 \%)$ & $267(10.7 \%)$ & $457(73.4 \%)$ \\
\hline NSTEMI & 2 & 39 & 19 & 195 & 37 & 193 & 58 & 427 \\
\hline \multirow[t]{3}{*}{ UAP } & 23 & 3 & 67 & 5 & 119 & 22 & 209 & 30 \\
\hline & \multicolumn{2}{|l|}{ Brisbane } & \multicolumn{2}{|l|}{ Christchurch } & \multicolumn{2}{|l|}{ Basel } & \multicolumn{2}{|l|}{ Combined } \\
\hline & $\begin{array}{l}\text { Ruled out } \\
525\end{array}$ & $\begin{array}{c}\text { Ruled in } \\
275\end{array}$ & $\begin{array}{l}\text { Ruled out } \\
276\end{array}$ & $\begin{array}{c}\text { Ruled in } \\
483\end{array}$ & $\begin{array}{l}\text { Ruled out } \\
993\end{array}$ & $\begin{array}{c}\text { Ruled in } \\
822\end{array}$ & $\begin{array}{l}\text { Ruled out } \\
1794\end{array}$ & $\begin{array}{l}\text { Ruled in } \\
1580\end{array}$ \\
\hline Ml at presentation & $2(0.4 \%)$ & $39(14.1 \%)$ & $4(1.5 \%)$ & $163(33.7 \%)$ & $1(0.1 \%)$ & $284(34.5 \%)$ & $7(0.4 \%)$ & 486 (30.8\%) \\
\hline 30-day MACE & $21(4.0 \%)$ & $50(18.2 \%)$ & $30(10.9 \%)$ & $200(41.4 \%)$ & $102(10.3 \%)$ & $371(45.1 \%)$ & $153(8.5 \%)$ & $621(39.3 \%)$ \\
\hline NSTEMI & 2 & 42 & 7 & 166 & 1 & 284 & 10 & 492 \\
\hline UAP & 19 & 8 & 23 & 34 & 101 & 87 & 143 & 129 \\
\hline
\end{tabular}

DG15, diagnostic guidance 15; MI, myocardial infarction; MACE, major adverse cardiac event; NSTEMI, non-ST elevation myocardial infarction; UAP, unstable angina pectoris; NICE, National Institute for Health and Care Excellence.

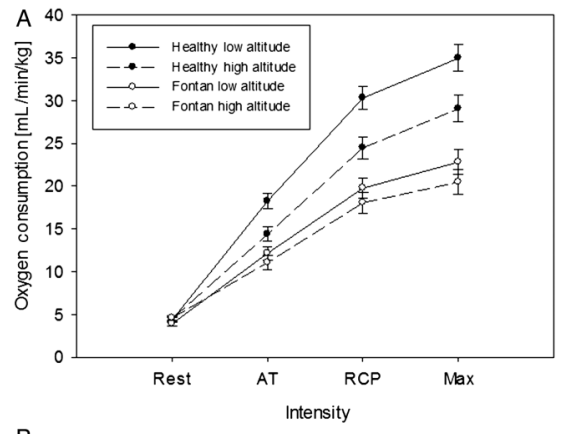

B

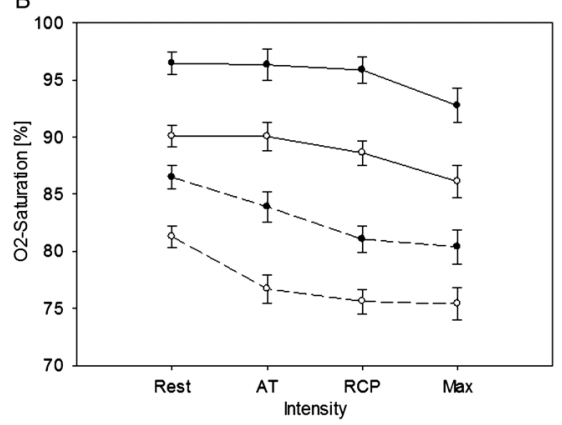

Figure 2 Cardiorespiratory parameters.

Figures show $(A)$ oxygen consumption and $(B)$ arterial oxygen saturation at rest, AT, RCP and maximal capacity during the cardiopulmonary exercise test at low and high altitude for the two study groups. AT, aerobic threshold; Max, peak exercise intensity; RCP, respiratory compensation point; Rest, resting baseline measurements on the bicycle. Values are presented as means and SEs.

study adds to our knowledge and importantly allows better counselling of patients planning alpine travel or commercial aeroplane flights".

The Education in Heart article in this issue (see page 1322) discusses cardiac

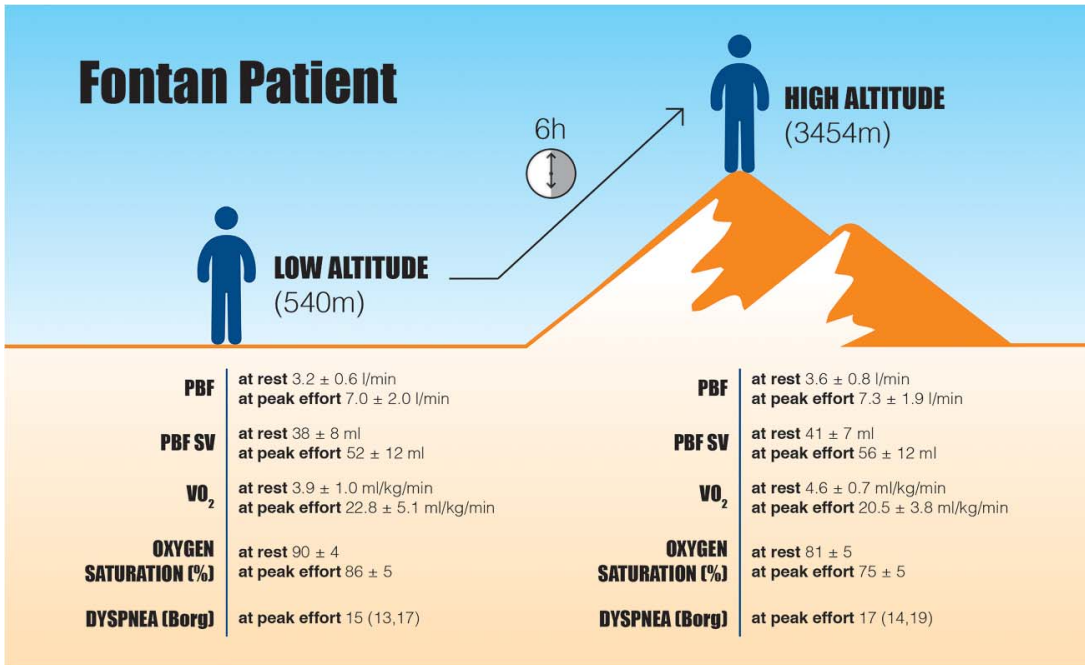

Figure 3 Effect of 6 hours high altitude exposure on Fontan patients showed no negative impact on pulmonary blood flow, exercise capacity and clinical symptoms. Values are means $\pm S D$ or median and interquartile range. PBF, pulmonary blood flow; PBF SV, pulmonary blood flow stroke volume; $\mathrm{VO}_{2}$, oxygen consumption.

consultation for patients who are undergoing non-cardiac surgery. Tools to estimate perioperative procedural risk and practical clinical algorithms are provided, with both the European Society of Cardiology and American College of Cardiology stepwise approaches summarized. Key messages include the principle that clinical tools should be used to assist in risk assessment, cardiac tests should only be performed if results will change management and the role of perioperative beta-blockers remains controversial whereas perioperative statins appear to be beneficial. "Communication and a team approach are essential to implement the consultant's recommendations and achieve the goal of medical optimisation and improved outcome."

The Image Challenge (see page 1295) asks you to identify the arrhythmia resulting in recurrent implanted defibrillator shocks in a 60 year old man.

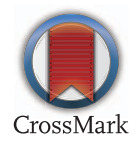

To cite Bradley SM, Otto CM. Heart 2016;102:1249-1250

Heart 2016;102:1249-1250.

doi:10.1136/heartjnl-2016-310271 